\title{
Factores determinantes del valor utilitarista en las aplicaciones móviles del sector moda
}

\author{
Key drivers of utilitarian value of mobile fashion apps \\ Miluska Murillo Zegarra*, Carla Ruíz Mafé \\ Universidad de Valencia, España
}

Recibido el 18 de julio de 2019; aceptado el 20 de abril de 2020

Disponible en Internet el: 12 de mayo de 2020

\section{Resumen}

El presente trabajo de investigación analiza los factores determinantes del valor utilitarista percibido en una aplicación de comercio móvil de productos de moda. Se propone y contrasta empíricamente un modelo de relaciones que analiza el impacto de las creencias ante la tecnología, las características del mensaje recibido a través de alertas y el marketing de permiso de las alertas (irritación y control percibido). El contraste de hipótesis realizado a partir de una muestra de 340 clientes reales en España, que compran productos de moda a través del móvil, evidencia que el valor utilitarista viene determinado por la utilidad percibida, el valor informativo de las alertas y el uso del marketing de permiso. La facilidad de uso incrementa la utilidad percibida, la personalización aumenta el valor informativo, y el control percibido disminuye la irritación.

Código JEL: M31, M31, L81

Palabras clave: Comercio móvil; Valor utilitarista; Valor informativo; Personalización; Irritación; Control percibido

\footnotetext{
*Autor para correspondencia. Correo electrónico: mimuze@alumni.uv.es (M. Murillo Zegarra).

La revisión por pares es responsabilidad de la Universidad Nacional Autónoma de México.
} 


\begin{abstract}
This paper identifies the key drivers of perceived utilitarian value when using a mobile fashion retail app. We analyze and test the impact of consumer beliefs regarding technology, the features of the mobile alerts messages and permission marketing variables (irritation and perceived control) related to the mobile alerts. A research model is tested using a sample of 340 mobile shoppers of fashion products in Spain. Results show that utilitarian value is determined by perceived usefulness, informativeness of the alerts and use of permission marketing. Ease of use increases the perceived usefulness, personalization increases the informativeness of the alerts and perceived control decreases consumer's irritation.
\end{abstract}

JEL Code: M31, L81

Keywords: Mobile commerce; Utilitarian value; Informativeness; Irritation; Perceived control

\title{
Introduction
}

Los smartphones (teléfonos móviles inteligentes) se han convertido en un potente medio de intercambio comercial. A nivel mundial el 55\% de los usuarios de internet ya realizan compras desde el móvil, con una tendencia que continuará en aumento y llegará a su auge conforme los nativos digitales vayan madurando como consumidores, llegando a suponer un $75 \%$ en 2021 (Ditrendia, 2019). En Europa, el porcentaje de ventas a través del móvil continúa creciendo, habiendo alcanzado un 44\% en 2018, ocupando el tercer puesto en el ranking mundial, por detrás de Oriente Medio y África (Ditrendia, 2019). España, con un $40 \%$ de usuarios de internet que ya compran desde el móvil, se encuentra aun ligeramente por debajo de la media mundial (Ditrendia, 2019). Esta realidad es una consecuencia del estilo de vida actual de los consumidores, donde los smartphones son un elemento indispensable, caracterizado por proporcionar información relevante en el lugar y momento en que se necesita. En consecuencia, las empresas también han otorgado a los smartphones un lugar predominante en sus estrategias de comunicación, aprovechando las características propias de este medio, como la ubicuidad, la localización, la conveniencia o la posibilidad de llegar al usuario de forma personalizada (Sanz et al., 2015, Clarke III, 2001). Así pues, dado que se trata de un canal con un importante potencial para realizar compras, y debido al explosivo crecimiento de las aplicaciones móviles, y que los patrones de comportamiento del comprador móvil son específicos y difieren de los del comprador online, el interés de los investigadores en este canal de marketing es creciente. Según el estudio de meta-análisis de Jun Jie Hew (2017), la tendencia en investigación sobre comercio móvil y sus especialidades ha aumentado constantemente, 
revelando que la investigación en esta área se encuentra ahora en su floreciente temporada. No obstante, existen todavía importantes lagunas en el estudio del comportamiento del usuario de aplicaciones móviles, siendo escasos los estudios previos realizados concretamente en la especialidad de compra a través del móvil. A título ilustrativo, cabe señalar los trabajos de Liu y Li (2019), Madan y Yadav (2018), Natarajan et al. (2018) y Verkijika (2018), que investigan sobre la intención de uso de la compra móvil. A pesar de sus ventajas, la naturaleza personal e intransferible de los dispositivos móviles también puede desencadenar actitudes negativas por parte del consumidor, que puede percibir las comunicaciones de marketing realizadas a través de las aplicaciones móviles como una intrusión en su privacidad (Kweon et al., 2012), disminuyendo así su valor percibido. Así pues, las empresas que utilicen un servicio de alertas en sus aplicaciones móviles deben hacer frente a importantes retos de cara a conseguir la aceptación e implicación del consumidor con sus mensajes. Una cuestión de investigación clave a analizar es el impacto de las comunicaciones intrusivas de marketing en el valor utilitarista percibido por los consumidores. El sector moda ha sido elegido como contexto para la presente investigación ya que en España ocupa la segunda posición en el ranking de productos más vendidos a través de los smartphones (45\%) después del sector de ocio (Ditrendia, 2019). Además, el sector de la moda es uno de los sectores más influyentes y de mayor crecimiento para las ventas a través de smartphones. Sin embargo, son escasos los estudios de comercio móvil aplicados al sector moda (Sun y Chi, 2018; Kim et al., 2009; Ko et al., 2009), siendo por tanto necesario un análisis más profundo. Desde el punto de vista de la gestión empresarial, un mayor conocimiento de los factores que conducen al incremento de la percepción del valor utilitarista percibido será beneficioso para las empresas del sector moda, porque podrán adaptar el contenido de sus aplicaciones móviles de manera que los clientes encuentren mejor cubiertas sus necesidades accediendo a una experiencia de mayor valor, incentivando de este modo la compra y recomendación.

Por lo anteriormente enunciado, se plantean los siguientes objetivos específicos para estainvestigación:

1. Estudiar la influencia de las características del mensaje (valor informativo, personalización), creencias del consumidor (facilidad de uso percibida y utilidad percibida) y de los antecedentes vinculados al marketing de permiso (irritación y control percibido) en el valor percibido utilitarista.

2. Realizar la propuesta y contraste de un modelo integrador de la influencia de las características del mensaje, creencias del consumidor, marketing de permiso, y valor utilitarista percibido.

3. Comprobar la validez y fiabilidad del modelo conceptual propuesto. 


\section{Revisión de la literatura y planteamiento de hipótesis}

El enfoque de la presente investigación ha tomado como base el Modelo de la Aceptación de la Tecnología (TAM) de Davis (1989). El modelo de aceptación de la tecnología (TAM) es uno de los mejores modelos probados para medir la aceptación (Zhang et al., 2012). Este modelo, desarrollado originalmente por Davis (1989), incluye la facilidad de uso percibida, la utilidad percibida, la actitud hacia el uso, la intención de uso conductual y el uso real del sistema. Donde la facilidad de uso y la utilidad percibidas son los determinantes actitudinales más importantes del uso real del sistema. Mientras que la actitud hacia el uso predice a la intención de uso de los usuarios, la misma que determina el uso real del Sistema.

\section{Valor percibido utilitarista}

El valor utilitarista del proceso de compra se refiere al valor relacionado con la consecución de un objetivo de compra. Los consumidores que perciben valor utilitarista en una aplicación móvil consideran que los beneficios en términos de ahorro, derivados de utilizar una aplicación móvil para realizar sus compras, son superiores en tiempo y esfuerzo, por lo que es de esperar que su predisposición a la compra será mayor. Así, desde el punto de vista utilitarista, a los consumidores les preocupa comprar productos de una forma eficiente y oportuna para conseguir sus objetivos con el mínimo de irritación (Childers et al., 2001). En el ámbito del comercio móvil, investigaciones previas han estudiado el valor utilitarista (Pipitwanichakarn, T., y Wongtada, N., 2019; Madan y Yadav, 2018; Khoi et al., 2018; Hew et al., 2015; Deng et al., 2014; Malik et al, 2013); siendo respaldado como una variable que guarda una estrecha relación con la adopción de la compra móvil (Natarajan et al., 2018; Natarajan et al., 2017; Liébana-Cabanillas et al.,2017; Wong et al., 2015) Asimismo, se han identificado un conjunto de beneficios utilitaristas asociados al acto de compra (facilidad de búsqueda de información, evaluación de alternativas y pago) que influyen en la intención de recompra a través de dispositivos móviles (Mahapatra et al., 2017).

\section{Antecedentes del valor percibido utilitarista}

\section{Creencias del consumidor: Utilidad percibida y Facilidad de uso}

En el contexto del presente estudio, la utilidad percibida se refiere al grado en que el consumidor cree que al utilizar la aplicación móvil para comprar productos de moda aumentará el rendimiento de su proceso de compra gracias al ahorro de tiempo y dinero asociado al uso de esa tecnología. La facilidad de uso percibida se define en el contexto de esta investigación como el grado en que el consumidor considera que el esfuerzo necesario para comprar pro- 
ductos de moda a través de una aplicación móvil es bajo, incluyendo el período de aprendizaje correspondiente. Varios estudios han demostrado, en el ámbito del comercio móvil, que la utilidad percibida y la facilidad de uso son importantes determinantes del valor percibido utilitarista (Natarajan et al., 2018, Liébana-Cabanillas et al., 2017). Por tanto, siguiendo la literatura revisada, se propone que, tanto la utilidad percibida como la facilidad de uso percibida en las aplicaciones móviles de productos de moda, influyen de forma positiva en el valor utilitarista percibido.

H1. La utilidad percibida en una aplicación de comercio móvil de productos de moda influye de forma positiva en el valor utilitarista percibido.

H2. La facilidad de uso percibida en una aplicación de comercio móvil de productos de moda influye de forma positiva en el valor utilitarista percibido.

Adicionalmente, la facilidad de uso percibida influye en la utilidad percibida, ya que la sencillez de un sistema puede mejorar el resultado (Davis, 1989; Davis et al., 1989), siendo una tecnología (aplicaciones móviles) percibida como más útil si es más fácil de usar. Por tanto, se plantea:

H2b. La facilidad de uso percibida en una aplicación de comercio móvil de productos de moda influye de forma positiva en la utilidad percibida.

\section{Características del mensaje: valor de la información y personalización}

El valor de la información se refiere a la habilidad para proveer de forma efectiva de información relevante (Oh y Xu, 2003). Así, la información entregada a los consumidores vía dispositivos móviles, según Siau y Shen (2003), necesita mostrar características cualitativas como exactitud, oportunidad, y utilidad para el consumidor; por lo tanto, lo que los consumidores esperan de los mensajes es lo que para ellos es relevante (Milne y Gordon, 1993). No es de extrañar que Chowdhury et al., (2006) encuentren que los consumidores no se sientan molestos si los anunciantes móviles presentan los anuncios con la información apropiada. Estudios recientes, en el campo de la publicidad móvil (Küster et al., 2017, Sanz et al., 2015) han demostrado la influencia del valor informativo en la actitud hacia el anuncio móvil. Por consiguiente, se propone:

H3. El valor de la información obtenido a través de las alertas publicitarias móviles influye de forma positiva en el valor utilitarista percibido de las aplicaciones de comercio móvil de productos de moda. 
La personalización es motivo de estudio en un contexto de marketing móvil, en tanto que los consumidores cada vez más esperan servicios a medida y basados en la localización. Estudios previos han demostrado que los clientes son receptivos ante la publicidad personalizada y relevante con su estilo de vida (DeZoysa, 2002). Conjuntamente con la hora y el lugar, los profesionales de marketing pueden orientar los anuncios móviles basados en las preferencias de los consumidores (Watson et al., 2002), lo que supone un aumento en el impacto de estos mensajes (Scharl et al., 2005). Es decir, la información enviada a través de las alertas publicitarias móviles podría ser más valiosa al estar personalizada. Además, los smartphones permiten construir perfiles de consumidores, monitorizando sus actividades y localización, permitiendo técnicas de personalización más sofisticadas basadas en las características sociodemográficas del consumidor, preferencias y contexto de la comunicación (Xu et al., 2008). En el contexto de la presente investigación, el análisis se ha realizado sobre la personalización de las alertas publicitarias móviles que se envían a los usuarios de la aplicación de comercio móvil de productos de moda. En el ámbito de la publicidad móvil, los estudios de Xu (2006), Sanz et al. (2015) y Küster et al., (2017) constatan la influencia de la personalización sobre la actitud hacia la publicidad móvil. Por tanto, se plantean las siguientes hipótesis:

H4. La personalización de las alertas publicitarias móviles influye de forma positiva en el valor utilitarista percibido de las aplicaciones de comercio móvil de productos de moda. H4b. La personalización de las alertas publicitarias móviles influye de forma positiva en el valor informativo de las aplicaciones de comercio móvil de productos de moda.

\section{Marketing de permiso: Irritación y control percibido}

La irritación del consumidor se refiere a los sentimientos de disgusto, incomodidad e ira causados por estímulos molestos como incidentes, mensajes o interacciones que pueden ir en contra de lo que un consumidor espera o anticipa en una situación particular (Ducoffe, 1996). En el contexto de la presente investigación, se estudia la irritación que podrían causar las alertas publicitarias móviles enviadas a los usuarios de la aplicación de comercio móvil de productos de moda. Los anuncios irritantes provocan molestia e impaciencia en los clientes (Ducoffe, 1995). Cuando la publicidad emplea técnicas que molestan, ofenden, insultan, o son demasiado manipuladoras, los consumidores tienden a percibirla como una influencia no deseada e irritante. La irritación puede conducir a una reducción general de la eficacia de la publicidad y el valor percibido por los consumidores (Aaker y Bruzzone, 1985; Luo, 2002). En el caso de la publicidad móvil, ésta puede proporcionar información excesiva y no relevante, que sea una distracción y que abrume al consumidor (Steward y Pavlov, 2002) y esto puede ser percibido como una intrusión en la privacidad del usuario móvil. Según 
el estudio de Ditrendia "Mobile en España y en el mundo" (2015), más de la mitad de los usuarios de marketing móvil españoles (52\%), no abren los anuncios de publicidad móvil porque los consideran molestos. Kim y Han (2014) evidencian la influencia negativa de la irritación causada por la publicidad móvil en el flujo de experiencia con la publicidad móvil. En este sentido, cuando la publicidad móvil contiene información que irrita al receptor, los consumidores pueden sentirse confusos y reaccionar negativamente (Kim y Han, 2014; Stewart y Pavlov, 2002; Xu, 2006). A veces las tácticas intrusivas que emplean los anunciantes a la hora de competir por la atención de los consumidores pueden ser molestas para el público (Zhang, 2000). Por consiguiente, se plantea:

H5. La irritación causada por las alertas publicitarias móviles influye de forma negativa en el valor utilitarista percibido de una aplicación de comercio móvil de productos de moda.

El control percibido describe la percepción individual sobre las habilidades o los recursos necesarios para asumir una decisión de comportamiento (Mathieson, 1991). En el contexto de la presente investigación, se refiere a la habilidad y recursos para modificar o cancelar el permiso para recibir las alertas publicitarias móviles. Se argumenta que la sensación de falta de control percibido puede ser un factor que impida a los consumidores participar en el marketing de los nuevos medios (Hoffman et al., 1999). La aplicación del control percibido a la tecnología móvil ofrece resultados contradictorios; algunos autores sugieren que el control percibido tiene poca asociación con las intenciones de recibir comunicaciones de marketing móvil (Karjaluoto y Alatalo, 2007; Merisavo et al., 2007; Venkatesh et al, 2003). Otros estudios apuntan que el control percibido es un factor determinante de la aceptación del marketing móvil (Barwise y Strong, 2002; Nysveen et al., 2005; Shankar et al., 2010). En el presente estudio se propone que control percibido influye de forma positiva sobre el valor utilitarista de una aplicación de comercio móvil de productos de moda. El consumidor que tenga las habilidades, recursos y oportunidades para modificar o cancelar las alertas recibidas, evitará la pérdida de tiempo y el esfuerzo dedicado a leer mensajes no deseados y por tanto percibirá una mayor eficiencia en sus decisiones de compra. Por tanto, se plantea la siguiente hipótesis:

H6. El control percibido sobre las alertas publicitarias móviles influye de forma positiva en el valor utilitarista percibido de una aplicación de comercio móvil de productos de moda.

No existe en la literatura académica ningún estudio que haya analizado los efectos del control percibido sobre la irritación en el contexto de las aplicaciones móviles. En otros medios interactivos como Internet, que ofrecen un alto grado de control al usuario sobre el medio, la intrusión, definida como la interrupción de los objetivos de los consumidores (Li et 
al., 2002), se ha detectado como un antecedente de los sentimientos de irritación (Edwards et al., 2002). El estudio de Sanz et al., (2011), evidencia que el control percibido disminuye la irritación ante los mensajes publicitarios del móvil en el colectivo de adolescentes. Teniendo en cuenta lo anterior se plantea:

H6b. El control percibido sobre las alertas publicitarias móviles influye de forma negativa en la irritación percibida de las mismas.

Las hipótesis planteadas conforman el siguiente modelo (ver figura 1).

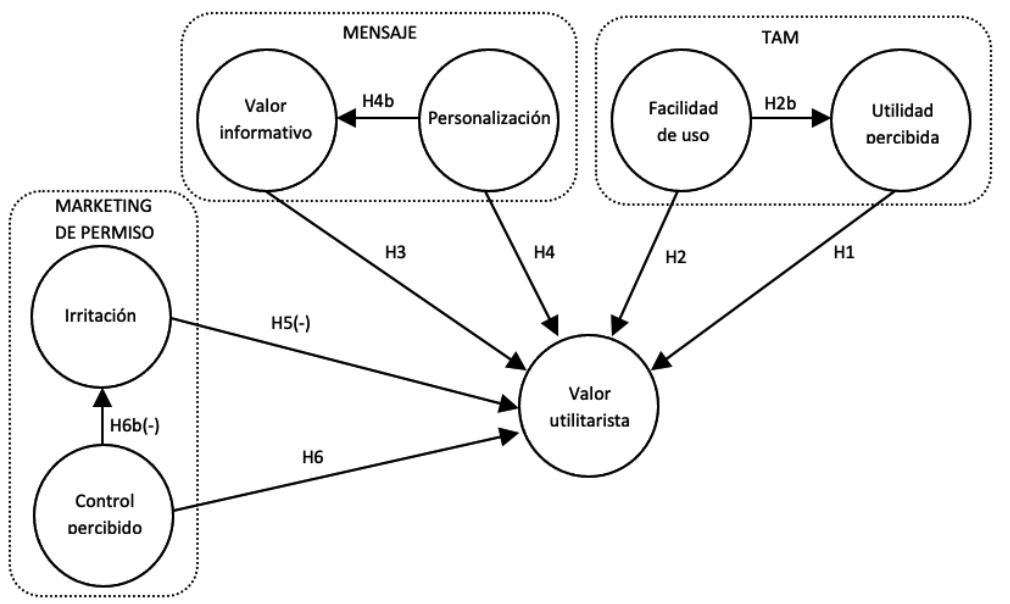

Figura 1. Modelo Conceptual

Fuente: Elaboración propia.

\section{Metodología}

Descripción de la muestra

Para responder a los objetivos planteados y contrastar las hipótesis planteadas, se ha desarrollado un estudio empírico de naturaleza causal, por medio de una encuesta online con cuestionario estructurado. El estudio empírico se ha llevado a cabo durante el mes de Julio de 2015. La captación de la muestra se realizó a través de cuatro envíos de un cuestionario estructurado online por correo electrónico a un total de 79.300 clientes, de una empresa multinacional española líder en comercio móvil en el sector moda, que realizan la navegación y compra, exclusivamente, a través de su aplicación móvil, lo que permite la medición del comporta- 
miento real de compra y recomendación. El procedimiento de elección de la muestra fue no probabilístico (conveniencia). Finalmente, tras un proceso de depuración para eliminar los cuestionarios incompletos o incorrectos, se obtuvieron 340 respuestas válidas.

La tabla 1 muestra las características del perfil de la muestra formada mayoritariamente por mujeres (70,5\%); cuya edad en su mayoría se ubica entre los rangos de 25 y $34(36,1 \%)$, y 35 y 44 años $(47,1 \%)$. Con un $66,1 \%$ con estudios superiores (universitarios, postgrado) siendo su principal ocupación la de trabajadores por cuenta ajena $(80,1 \%)$. En cuanto al comportamiento de compra (Ver tabla 2), el 65\% tiene más de 1 año de antigüedad como comprador, siendo la frecuencia de compra mayor a 3 veces en el $43,5 \%$ de los casos (heavy-shoppers). En cuanto a los productos comprados, destacan la ropa $(79,9 \%)$ y el calzado $(77,7 \%)$, seguidos por los accesorios $(49,6 \%)$.

Tabla 1

Características de la muestra

\begin{tabular}{ccc}
\hline Característica & Categoría & Porcentaje vertical \\
\hline \multirow{2}{*}{ Sexo } & Hombre & $29,50 \%$ \\
& Mujer & $70,50 \%$ \\
Edad (años) & $15-24$ & $3,60 \%$ \\
& $25-34$ & $36,10 \%$ \\
& $35-44$ & $47,10 \%$ \\
Nivel de estudios & $45-54$ & $11,30 \%$ \\
& Más de 55 & $1,90 \%$ \\
& Primarios & $2,20 \%$ \\
& Secundarios & $11,00 \%$ \\
& Formación profesional & $20,70 \%$ \\
& Universitarios & $52,60 \%$ \\
& Postgrado & $13,50 \%$ \\
& Cuenta ajena & $80,10 \%$ \\
& Cuenta propia & $8,60 \%$ \\
& Desempleado & $5,20 \%$ \\
& Estudiante & $2,80 \%$ \\
& Pensionista & $0,60 \%$ \\
& Labores del hogar & $2,80 \%$ \\
\hline
\end{tabular}

Fuente: Elaboración propia 
Tabla 2

Descripción de la muestra según comportamiento de compra

\begin{tabular}{ccc}
\hline Característica & Categoría & Porcentaje vertical \\
\hline & Menos de 6 meses & $21,20 \%$ \\
Antigüedad como & De 6 meses a 1 año & $13,80 \%$ \\
comprador & De 1 a 2 años & $29,20 \%$ \\
& Más de 2 años & $35,80 \%$ \\
Frecuencia de compra & 1 vez & $15,70 \%$ \\
(Durante el último año) & 1 - 3 veces & $40,80 \%$ \\
& 4 - 7 veces & $31,10 \%$ \\
& Más de 7 veces & $12,40 \%$ \\
& Ropa & $79,90 \%$ \\
Productos comprados & Calzado & $77,70 \%$ \\
& Accesorios & $49,60 \%$ \\
& Ropa deportiva & $30,30 \%$ \\
\hline
\end{tabular}

Fuente: Elaboración propia

\section{Medición}

El instrumento de captación de información utilizado es una encuesta online, elaborada tomando como base las escalas existentes encontradas en la literatura, utilizando una escala Likert de 7 puntos ( 1 "nada de acuerdo, 7 "totalmente de acuerdo"). A continuación, se detallan las escalas utilizadas para la estimación de las variables objeto de estudio. El valor utilitarista percibido se ha medido siguiendo la escala propuesta por Sirdeshmukh et al. (2002), y posteriormente aplicadas por Kim y Han (2011) y Kim y Oh (2011). Continuando con los antecedentes del valor utilitarista, la utilidad percibida y facilidad de uso han sido medidos adaptando la escala desarrollada por Davis (1989) y Davis et al., (1989). La personalización ha sido medida adaptando la escala utilizada por Xu (2006) y Merisavo et al. (2007), y el valor informativo, adaptando la escala propuesta por Wang y Sun, (2010) y aplicada por Lui et al., (2012). En cuanto a las variables de marketing del permiso, por un lado, la irritación se ha medido adaptando la escala propuesta por Lee et al., (2006), y el control percibido se ha medido adaptando la escala propuesta por Vogt (1997) y aplicada posteriormente por Kautonen y Kohtamaki,(2006). En el apéndice 1 se expone cómo se han adaptado las escalas anteriormente mencionadas en la presente investigación. 


\section{Validación del instrumento de medida}

Con el fin de asegurar la validez y fiabilidad de los datos obtenidos, se ha llevado a cabo un análisis Factorial confirmatorio (AFC) ejecutado en el programa EQS 6.1. Los resultados no mostraron problemas de validez convergente, ya que el AFC mostró un ajuste del modelo satisfactorio (Chi cuadrado de 519,501 con 231 grados de libertad, NFI=0,935; NNFI=0,955, $\mathrm{CFI}=0,963$ e IFI=0,963; RMSEA $=0,061$ ), y todas las cargas factoriales fueron significativas y superiores a 0.6 (Bagozzi y Baumgartner, 1994; Bagozzi y Yi, 1988). Tampoco hubo problemas de fiabilidad, tanto atendiendo al criterio del $\alpha$ de Cronbach (Cronbach, 1951), como del de la fiabilidad compuesta (superior siempre al punto de corte de .70) y la varianza extraída promedio (superior siempre al punto de corte de .50). Ver tabla 3.

Para evaluar la validez discriminante se utilizó: (a) la aproximación sugerida por Anderson y Gerbing (1988), denominado test del intervalo de confianza, que implica calcular un intervalo de confianza de +/-2 errores estándar entre la correlación de los factores, determinando la validez si el intervalo no incluye el valor 1; y (b) el test de la varianza extraída (Fornell y Larcker, 1981), verificando que el cuadrado de la covarianza de cada par de factores sea menor que la varianza extraída de cada uno de esos factores. Como puede observarse en la tabla 4, estas condiciones se cumplieron para cada uno de los factores, constatando la validez discriminante del modelo de medida.

\section{Análisis y discusión de resultados}

Con el fin de testar las hipótesis propuestas, se ha utilizado el método de Modelización Estructural basada en covarianzas, ejecutándose en el programa EQS 6.1. Este método estructural arrojó un ajuste satisfactorio del modelo: Chi cuadrado de 580,639 con 240 grados de libertad, NFI $=0,927 \mathrm{NNFI}=0,949, \mathrm{CFI}=0,956$ e IFI $=0,956$. El RMSEA $=0,065$ muestra un ajuste aceptable al ser menor de 0,8 . La tabla 5 , recoge los valores de las cargas estandarizadas de las relaciones estructurales propuestas, indicando sus correspondientes niveles de significatividad deducidos de sus respectivos valores estadísticos "t" asociados. 
Tabla 3

Resultados del AFC y propiedades psicométricas del modelo de medida

\begin{tabular}{|c|c|c|c|c|c|c|c|}
\hline Concepto & Items & Carga & $\begin{array}{c}\text { Carga } \\
\text { estandarizada }\end{array}$ & Valor-t & $\begin{array}{l}\text { Cron- } \\
\text { bach }\end{array}$ & IFC & AVE \\
\hline \multirow{4}{*}{ Utilidad percibida } & UP1 & 1,097 & 0,878 & 20,388 & \multirow{4}{*}{0,918} & \multirow{4}{*}{0,920} & \multirow{4}{*}{0,742} \\
\hline & UP2 & 1,111 & 0,890 & 20,799 & & & \\
\hline & UP3 & 1,123 & 0,899 & 21,138 & & & \\
\hline & UP4 & 1,123 & 0,761 & 16,306 & & & \\
\hline \multirow{4}{*}{ Facilidad de uso } & FU1 & 0,925 & 0,873 & 20,108 & \multirow{4}{*}{0,913} & \multirow{4}{*}{0,919} & \multirow{4}{*}{0,742} \\
\hline & FU2 & 0,982 & 0,970 & 24,128 & & & \\
\hline & FU3 & 0,826 & 0,690 & 14,988 & & & \\
\hline & FU4 & 0,946 & 0,871 & 20,156 & & & \\
\hline \multirow{4}{*}{ Valor utilitarista } & VU1 & 0,812 & 0,705 & 14,947 & \multirow{4}{*}{0,897} & \multirow{4}{*}{0,899} & \multirow{4}{*}{0,690} \\
\hline & VU2 & 0,928 & 0,824 & 18,372 & & & \\
\hline & VU3 & 1,075 & 0,897 & 20,709 & & & \\
\hline & VU4 & 1,080 & 0,878 & 19,685 & & & \\
\hline \multirow{4}{*}{ Valor informativo } & VI1 & 1,493 & 0,893 & 21,087 & \multirow{4}{*}{0,962} & \multirow{4}{*}{0,961} & \multirow{4}{*}{0,861} \\
\hline & VI2 & 1,516 & 0,921 & 22,085 & & & \\
\hline & VI3 & 1,579 & 0,957 & 23,813 & & & \\
\hline & VI4 & 1,505 & 0,940 & 23,021 & & & \\
\hline \multirow{3}{*}{ Control percibido } & $\mathrm{CP} 1$ & 1,592 & 0,916 & 21,592 & \multirow{3}{*}{0,887} & \multirow{3}{*}{0,895} & \multirow{3}{*}{0,742} \\
\hline & $\mathrm{CP} 2$ & 1,649 & 0,928 & 22,017 & & & \\
\hline & $\mathrm{CP} 3$ & 1,297 & 0,720 & 15,081 & & & \\
\hline \multirow{2}{*}{ Personalización } & PE1 & 1,489 & 0,874 & 19,521 & \multirow{2}{*}{0,893} & \multirow{2}{*}{0,894} & \multirow{2}{*}{0,808} \\
\hline & PE2 & 1,487 & 0,917 & 20,961 & & & \\
\hline \multirow{3}{*}{ Irritación } & IR1 & 1,321 & 0,760 & 16,293 & \multirow{3}{*}{0,908} & \multirow{3}{*}{0,913} & \multirow{3}{*}{0,779} \\
\hline & IR2 & 1,670 & 0,918 & 21,680 & & & \\
\hline & IR3 & 1,698 & 0,958 & 23,072 & & & \\
\hline$* *=p<0,01 ; *=p$ & & & & & & & \\
\hline
\end{tabular}

Fuente: Elaboración propia. 
Tabla 4

Validez discriminante

\begin{tabular}{|c|c|c|c|c|c|c|c|}
\hline & UP & FU & VU & VI & VP & PE & IR \\
\hline Utilidad percibida & 0,861 & $\begin{array}{c}(0,741 ; \\
0,837)\end{array}$ & $\begin{array}{c}(0,694 \\
0,810)\end{array}$ & $\begin{array}{l}0,210 \\
0,418)\end{array}$ & $\begin{array}{c}(0,219 ; \\
0,431)\end{array}$ & $\begin{array}{c}(0,259 ; \\
0,467)\end{array}$ & $(-0,311 ;-0,087)$ \\
\hline Facilidad de uso & 0,789 & 0,862 & $\begin{array}{c}(0,559 ; \\
0,707)\end{array}$ & $\begin{array}{c}(0,141 ; \\
0,353)\end{array}$ & $\begin{array}{c}(0,176 ; \\
0,388)\end{array}$ & $\begin{array}{c}(0,145 \\
0,365)\end{array}$ & $(-0,295 ;-0,075)$ \\
\hline Valor utilitarista & 0,752 & 0,633 & 0,831 & $\begin{array}{c}(0,352 ; \\
0,540)\end{array}$ & $\begin{array}{c}(0,314 ; \\
0,514)\end{array}$ & $\begin{array}{c}(0,322 ; \\
0,522)\end{array}$ & $(-0,493 ;-0,293)$ \\
\hline Valor informativo & 0,314 & 0,247 & 0,446 & 0,928 & $\begin{array}{c}(0,516 \\
0,668)\end{array}$ & $\begin{array}{c}(0,533 ; \\
0,685)\end{array}$ & $(-0,601 ;-0,433)$ \\
\hline Control percibido & 0,325 & 0,282 & 0,414 & 0,592 & 0,862 & $\begin{array}{c}(0,714 \\
0,826)\end{array}$ & $(-0,636 ;-0,468)$ \\
\hline Personalización & 0,363 & 0,255 & 0,422 & 0,609 & 0,770 & 0,899 & $(-0,577 ;-0,393)$ \\
\hline Irritación & $-0,199$ & $-0,185$ & $-0,393$ & $-0,517$ & $-0,552$ & $-0,485$ & 0,882 \\
\hline
\end{tabular}

Fuente: Elaboración propia.

Tabla 5

Modelo de Ecuaciones Estructurales. Contraste de las Hipótesis

\begin{tabular}{lcccc}
\hline & Hipótesis a contrastar & Valor-t & $\begin{array}{c}\text { Carga estanda- } \\
\text { rizada }\end{array}$ & Confirmación \\
\hline H1 & Utilidad percibida - Valor utilitarista & 7,425 & $0,602^{* *}$ & Aceptada \\
H2 & Facilidad de uso - Valor utilitarista & NS & & Rechazada \\
H2b & Facilidad de uso - Utilidad percibida & 15,968 & $0,792^{* *}$ & Aceptada \\
H3 & Valor informativo - Valor utilitarista & 2,742 & $0,149^{* *}$ & Aceptada \\
H4 & Personalización - Valor utilitarista & NS & & Rechazada \\
H4b & Personalización - Valor informativo & 11,962 & $0,635^{* *}$ & Aceptada \\
H5(-) & Irritación - Valor utilitarista & $-3,511$ & $-0,179 * *$ & Aceptada \\
H6 & Control percibido - Valor utilitarista & NS & & Rechazada \\
H6b(-) & Control percibido - Irritación & $-9,837$ & $-0,561 * *$ & Aceptada \\
\hline$* *=\mathrm{p}<0,01 * *<0,05$ & & &
\end{tabular}

$* *=\mathrm{p}<0,01 ; *<0,05$

NS: No satisfactorio

Fuente: Elaboración propia. 


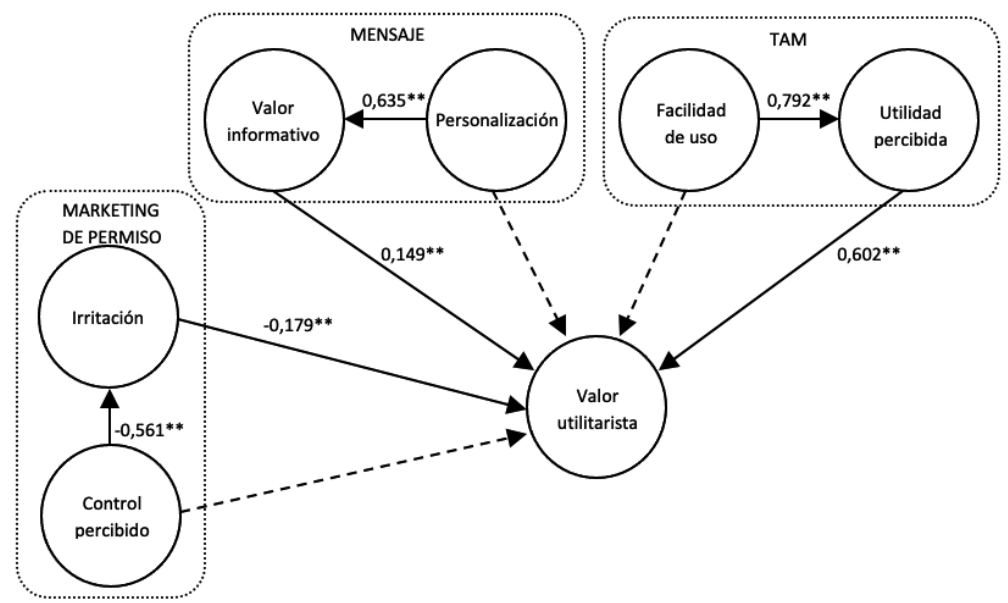

Figura 2. Síntesis de Resultados

Fuente: Elaboración propia.

A continuación, se realiza la discusión de resultados y el contraste de hipótesis de acuerdo a los objetivos propuestos.

En relación a los antecedentes del valor utilitarista, se confirma que la utilidad percibida influye positivamente en el valor utilitarista (H1 aceptada; $\left.\beta=0,602^{* *}\right)$, lo que indica que el usuario valora el grado en el que la aplicación móvil mejora su desempeño en la compra de productos de moda (Davis, 1989). Mientras que la facilidad de uso influye indirectamente sobre el valor utilitarista a través de la utilidad percibida (H2b aceptada; $\beta=0,792 * *)$, con una carga muy significativa, confirmando lo establecido en el modelo TAM (Davis, 1989; Davis et al., 1989). La simplicidad de uso de la aplicación móvil durante el proceso de compra de productos de moda influye en la percepción de la eficiencia asociada al proceso de compra, incrementando la percepción de su utilidad. Por tanto, se complementan hallazgos previos (Ruiz et al., 2010) vinculados al modelo de la aceptación de la tecnología (TAM) de Davis (1989), demostrando que las creencias vinculadas a la tecnología (utilidad y facilidad de uso), en el contexto objeto de estudio, son determinantes claves del valor utilitarista percibido en la aplicación de comercio móvil. Con respecto a la relación entre las características del mensaje y el valor utilitarista, los resultados muestran que el valor informativo de las alertas publicitarias móviles influye positivamente en el valor utilitarista de la aplicación móvil (H3 aceptada; $\left.\beta=0,149^{* *}\right)$. Mientras que la personalización, aunque no influye directamente sobre el valor utilitarista (H4 rechazada), si influye indirectamente sobre el valor utilitarista a través del valor informativo ( $\mathrm{H} 4 \mathrm{~b}$ aceptada; $\beta=0,635^{* *}$ ). El propósito de la personalización es incrementar la relevancia de la publicidad para los consumidores, mejorando su receptividad hacia los mensajes publicitarios, siendo una característica intrínseca y diferencial de la 
publicidad móvil (Xu et al., 2008). Por consiguiente, es posible que los usuarios consideren que todas las alertas deben estar ajustadas a sus preferencias antes de enviarlas, de forma que es una característica que no les aporta valor añadido adicional. En cuanto a la relación entre las variables del marketing de permiso y el valor utilitarista, se observa que la irritación influye significativamente y de forma negativa sobre el valor utilitarista (H5 (-) aceptada; $\beta=-0,179 * *)$. Este resultado implica que el efecto de la irritación en el valor utilitarista percibido que ha sido comprobado en el contexto de la publicidad móvil (Kim y Han, 2014) puede también ser extendido al contexto específico de las alertas enviadas por aplicaciones móviles en el sector moda. No obstante, no se encontró relación entre el control percibido y el valor utilitarista, siendo rechazada esta hipótesis (H6 rechazada). A su vez, el control percibido influye muy significativamente y de forma negativa sobre la irritación (H6b (-) aceptada; -0,561**), lo que señala que, a mayor control percibido por el usuario, disminuye la sensación de irritación generada por excesiva frecuencia de exposición, etc. Esta relación apenas ha sido contrastada en la literatura en marketing móvil por lo que se complementa la literatura de marketing de permiso.

\section{Conclusiones}

Esta investigación contribuye a la literatura académica sobre valor utilitarista percibido, aportando la propuesta y contraste de un modelo integrador de la influencia de las características del mensaje, creencias del consumidor y marketing de permiso en el valor utilitarista percibido. Tal y como indican Boksberger y Melsen, (2011) es necesario abordar el estudio específico de cada una de las dimensiones del valor utilitarista percibido así como su interacción con otros constructos de marketing. Así, el modelo planteado aporta una visión diferente de relaciones entre variables que puede ser útil a las propuestas realizadas con anterioridad en la literatura específica sobre factores utilitaristas y su relación con el valor percibido en los entornos móviles en el sector moda, como por ejemplo el trabajo de Ko et al. (2009) donde se estudia la influencia de la facilidad de uso percibida, la utilidad percibida, la conectividad instantánea y el disfrute sobre el valor percibido. En la presente investigación, se plantea la influencia no solo de la facilidad de uso percibida y la utilidad percibida, sino también del valor informativo, personalización, control percibido y la irritación, sobre la dimensión utilitarista del valor percibido. Asimismo, al proponer el estudio de la irritación y del control percibido en relación a las alertas publicitaria móviles, donde el control percibido es la variable que reduce la irritación producida por las mismas, la presente investigación podría complementar trabajos como el de Kim y Han (2014) que analizó la influencia negativa de la irritación en el flujo de experiencia con la publicidad móvil. La aplicación del estudio al contexto específico del sector moda, constituye otra contribución, dadas las escasas investigaciones aplicadas al 
mismo a pesar de su potencial en el comercio móvil. Desde el punto de vista empresarial, se derivan también implicaciones de carácter gerencial, relevantes para las empresas del sector moda que utilizan el móvil como canal de venta, de cara a reforzar los principales factores que influyen en el valor utilitarista percibido. Para incrementar el valor utilitarista hay que trabajar las variables que, a su vez influyen en el propio valor utilitarista: el factor con mayor influencia en el valor utilitarista es la utilidad percibida, que a su vez está influido fuertemente por la facilidad de uso. En este sentido, los usuarios cada vez exigen menor esfuerzo, mayor rapidez y eficacia en el uso de la aplicación. Por tanto, una estrategia para ofrecer mayor utilidad es garantizar que todos los pasos involucrados en el flujo de compra a través de la aplicación móvil (navegación por los productos, añadir los productos al carrito, iniciar el pedido, confirmación de la información de envío, y pago) se realicen de forma simple (con el mínimo número de tareas imprescindibles), flexible, y con una adaptabilidad eficiente y dinámica a las funcionalidades de las diferentes plataformas. Por tanto, debe identificarse también cualquier elemento en la funcionalidad de los distintos dispositivos que den lugar al abandono de la aplicación. El valor utilitarista también puede verse incrementado a través del valor informativo, que en el contexto del presente estudio se refiere concretamente a la información recibida a través de las alertas publicitarias móviles, por ejemplo, para dar a conocer nuevos productos disponibles, descuentos e incentivos; una estrategia que capta la atención del usuario y le lleva a la tienda móvil. Asimismo, el valor informativo está fuertemente influido por la personalización. En este sentido los usuarios esperan que los mensajes sean lo que para ellos es relevante. Por tanto, la recomendación para las empresas del sector, en este aspecto, es ofrecer información oportuna, exacta y que le sea útil al usuario, con el correspondiente elemento de personalización. Con este fin se podría llevar a cabo un estudio cualitativo, así como encuestas periódicas que indiquen qué información le interesa recibir al usuario a través de alertas, así como sobre la propia forma de la alerta, por ejemplo enviar alertas con un icono diferente intentando clasificarlas, según la información enviada (promoción que está a punto de terminar, nuevos productos, incentivos por la compra de una cantidad mínima, etc.) de modo que el usuario identifique el símbolo del icono, haciéndole más fácil reconocer la relevancia de la información, y según esto, la premura de hacer clic en la alerta instantáneamente o que recuerde que debe hacerlo pronto, para no perder por ejemplo una promoción que está a punto de terminar. Otro aspecto relevante que incrementa el valor utilitarista es la disminución de la irritación que podrían causar las alertas enviadas a los usuarios. Una de las claves, es proporcionar al usuario mayor control sobre el envío de las alertas. Por tanto, la recomendación es, por un lado, encontrar el balance adecuado en el envío de las alertas publicitarias móviles, de modo que no provoque la irritación de los usuarios, para lo cual se debe encontrar cuál es el umbral de tolerancia. Por otro lado, hacer sentir al usuario que tiene el control sobre el envío de alertas, a través de tácticas de marketing de 
permiso, por ejemplo, recordar al usuario de forma periódica que puede cambiar sus preferencias sobre el envío de alertas, a menos que estén satisfechos con el valor de la información recibida. Sería conveniente también que la aplicación implemente funciones que le permitan al usuario poder expresar sus opiniones, de forma sencilla y efectiva. Es necesario también mencionar algunas limitaciones de la investigación y algunas sugerencias para futuros trabajos. Este estudio se ha centrado en una empresa, sector y canal de compra determinado (la compra de productos de moda a través aplicaciones de comercio móvil), lo que debe tenerse en cuenta en la extensión de las conclusiones obtenidas al sector moda en general o a otros formatos del marketing móvil. Sugerimos, en consecuencia, aplicar las relaciones del modelo propuesto a una muestra representativa de compradores a través de aplicaciones móviles. También, la rapidez de la evolución del entorno electrónico aconseja repetir el estudio de manera periódica ya que las conclusiones pueden variar rápidamente como consecuencia de la propia naturaleza del contexto en el que se ha desarrollado esta investigación. Otra posible limitación es que el estudio se ha centrado en la medición de intenciones, que no siempre se convierten en comportamientos. Por lo anteriormente expuesto, se recomienda utilizar la técnica de experimentación para evaluar el comportamiento real de compra.

\section{Agradecimientos}

Las autoras agradecen el apoyo del proyecto UV-INV-AE19-1212255

\section{Referencias}

Aaker, D. A. \& Bruzzone, D. E. (1985). Causes of irritation in advertising. The Journal of Marketing, 49(2), $47-57$. https://doi.org/10.1177/002224298504900204

Ajzen, I. (1985). From intentions to actions: a theory of planned behavior. En Kuhl, J., Bechmann, J. (Eds.) SSSP Springer Series in Social Psychology. (pp. 11-39). Springer, Berlin, Heidelberg. https://doi.org/10.1007/9783-642-69746-3_2

Anderson, J. C. \& Gerbing, D. W. (1988). Structural equation modeling in practice: A review and recommended two-step approach. Psychological Bulletin, 103(3), 411. https://doi.org/10.1037/0033-2909.103.3.411

Bagozzi, R. P. \& Baumgartner, H. (1994). The Evaluation of Structural Equation Models and Hypothesis Testing. In Richard P. Bagozzi (Ed.). Principles of Marketing Research, MA: Blackwell, Cambridge, 386-422.

Baggozi, R. P. \& Yi, Y. (1988). On the evaluation of structural equation models. Journal of the Academy of Marketing Science, 16(1), 74-94. http://dx.doi.org/10.1007/BF02723327

Barwise, P. \& Strong, C. (2002). Permission-based mobile advertising. Journal of Interactive Marketing, 16(1), 14-24. https://doi.org/10.1002/dir.10000

Boksberger, P. and Melsen, L. (2011), "Perceived value: a critical examination of definitions, concepts and measures for the service industry", Journal of Services Marketing, 25 (3), 229-240, https://doi. org/10.1108/08876041111129209.

Childers T. L., Carr C. L. \& Peck J. Carson S. (2001). Hedonic and utilitarian motivations for online retail shopping behavior. Journal of Retailing, 77(4), 511-525. https://doi.org/10.1016/S0022-4359(01)00056-2 
Chowdhury, H. K., Parvin, N. \& Weitenberner, C., Becker, M. (2006). Consumer attitude toward mobile advertising in an emerging market: An empirical study. International Journal of Mobile Marketing, 1(2), 33-41. Disponible en: http://citeseerx.ist.psu.edu/viewdoc/download?doi=10.1.1.466.7036\&rep=rep1\&type=pdf Consultado: 20/01/2019.

Clarke III, I. (2001). Emerging value propositions for m-commerce. Journal of business strategies, 18(2), 133. Disponible en: https://search.proquest.com/docview/204381311?pq-origsite=gscholar Consultado: 20/01/2019.

Cronbach, L. J. (1951). Coefficient alpha and the internal structure of tests. Psychometrika, 16(3), 297-334. https:// doi.org/10.1007/BF02310555

Davis, F. D. (1989). Perceived usefulness, perceived ease of use, and user acceptance of information technology. MIS Quarterly, 13(3), 319-341. https://doi.org/10.2307/249008

Davis, F. D., Bagozzy, R. \& Warshaw, P. R. (1989). User acceptance of computer technology: a comparison of two theoretical models. Management Science, 35(8), 982-1003. https://doi.org/10.1287/mnsc.35.8.982

Deng, Z., Mo, X. \& Liu, S. (2014). Comparison of the middle-aged and older users' adoption of mobile health services in China. International Journal of Medical Informatics, 83(3), 210-224. https://doi.org/10.1016/j.ijmedinf.2013.12.002

DeZoysa, S. (2002). Mobile advertising needs to get personal. Telecommunications International, 36(2), 8. Disponible en: https://www.researchgate.net/publication/285650940_Mobile_advertising_needs_to_get_personal Consultado: 20/01/2019.

Ditrendia (2019). Informe Mobile en España y en el Mundo 2019. Disponible en https://ditrendia.es/informe-mobile-espana-mundo-2019/ Consultado: 20/04/2019.

Ditrendia (2015). Informe Mobile en España y en el mundo 2015. Disponible en https://ditrendia.es/wp-content/uploads/2015/07/Ditrendia-Informe-Mobile-en-Espa\%C3\%B1a-y-en-el-Mundo-2015.pdf Consultado: 20/04/2019.

Ducoffe, R. H. (1995). How consumers assess the value of advertising. Journal of Current Issues and Research in Advertising, 17(1), 1-18. https://doi.org/10.1080/10641734.1995.10505022

Ducoffe, R. H. (1996). Advertising value and advertising on the Web. Journal of Advertising Research, 36 (5), 21-35.

Edwards, S. M., Li, H. \& Lee, J. H. (2002). Forced exposure and psychological reactance: Antecedents and consequences of the perceived intrusiveness of pop-up ads. Journal of Advertising, 31(3), 83-95. https://doi.org/10. 1080/00913367.2002.10673678

Fornell, C. \& Larcker, D. F. (1981). Evaluating structural equation models with unobservable variables and measurement error. Journal of Marketing Research, 18, 39-50. https://doi.org/10.1177/002224378101800104

Hew, J. J. (2017). Hall of fame for mobile commerce and its applications: A bibliometric evaluation of a decade and a half (2000-2015). Telematics and Informatics, 34(1), 43-66. https://doi.org/10.1016/j.tele.2016.04.003

Hew, J. J., Lee, V. H., Ooi, K. B. \& Wei, J. (2015). What catalyses mobile apps usage intention: an empirical analysis. Industrial Management and Data Systems, 115(7), 1269-1291. https://doi.org/10.1108/IMDS-012015-0028

Hoffman, D. L., Novak, T. P. \& Peralta, M. (1999). Building consumer trust online. Communications of the ACM, 42(4), 80-85. https://doi.org/10.1145/299157.299175

Karjaluoto, H. \& Alatalo, T. (2007). Consumers' attitudes towards and intention to participate in mobile marketing. International Journal of Services Technology and Management, 8(2-3), 155-173. https://doi.org/10.1504/ IJSTM.2007.012866

Kautonen, T. \& Kohtamäki, M. (2006). Endogenous and exogenous determinants of trust in inter-firm relations: a conceptual analysis based on institutional economics. Finnish Journal of Business Economics, 55(3), 277-295. Disponible en: https://ssrn.com/abstract=2271628 Consultado: 20/02/2019. 
Khoi, N. H., Tuu, H. H. \& Olsen, S. O. (2018). The role of perceived values in explaining Vietnamese consumers' attitude and intention to adopt mobile commerce. Asia Pacific Journal of Marketing and Logistics. https://doi. org/10.1108/APJML-11-2017-0301

Kim, J., Ma, Y. J. \& Park, J. (2009). Are US consumers ready to adopt mobile technology for fashion goods? Journal of Fashion Marketing and Management: An International Journal. 13(2), 215-230. https://doi. org/10.1108/13612020910957725

Kim, Y. J. \& Han, J. (2014). Why smartphone advertising attracts customers: A model of web advertising, flow, and personalization. Computers in Human Behavior, 33, 256-269. https://doi.org/10.1016/j.chb.2014.01.015

Kim, B. \& Han, I. (2011). The role of utilitarian and hedonic values and their antecedents in a mobile data service environment. Expert Systems with Applications, 38(3), 2311-2318. https://doi.org/10.1016/j.eswa.2010.08.019

Kim, B. \& Oh, J. (2011). The difference of determinants of acceptance and continuance of mobile data services: A value perspective, Expert Systems with Applications, 38(3), 1798-1804. https://doi.org/10.1016/j. eswa.2010.07.107

Ko, E., Kim, E. Y. \& Lee, E. K. (2009). Modeling consumer adoption of mobile shopping for fashion products in Korea. Psychology \& Marketing, 26(7), 669-687. https://doi.org/10.1002/mar.20294

Küster, I., Ruiz, C. \& Damian, C. (2017). ¿Es efectiva la publicidad móvil?. Revista de Ciencias Sociales, 23(1), 22-33. Disponible en: https://www.redalyc.org/pdf/280/28056725003.pdf Consultado: 20/01/2019

Kweon, H., Zhang, D. \& Zhou, L. (2012). User privacy in mobile advertising. AMCIS 2012 Proceedings, 1. Disponible en: https://aisel.aisnet.org/amcis2012/proceedings/HCIStudies/1/ Consultado: 20/01/2019

Lee, S.F., Tsai, Y.C. \& Jih, W.J. (2006). An Empirical Examination of Customer Perceptions of Mobile Advertising. Information Resources Management Journal, 19(4), 39-55. http://dx.doi.org/10.4018/irmj.2006100103

Li, H., Edwards, S. M. \& Lee, J. H. (2002). Measuring the intrusiveness of advertisements: Scale development and validation. Journal of Advertising, 31(2), 37-47. http://dx.doi.org/10.1080/00913367.2002.10673665

Liébana-Cabanillas, F, Marinkovic, V. \& Kalinic, Z. (2017). A SEM-neural network approach for predicting antecedents of m-commerce acceptance. International Journal of Information Management, 37(2), 14-24. https:// doi.org/10.1016/j.ijinfomgt.2016.10.008

Liu, D. \& Li, M. (2019). Exploring new factors affecting purchase intention of mobile commerce: trust and social benefit as mediators. International Journal of Mobile Communications, 17(1), 108-125. http://dx.doi. org/10.1504/IJMC.2019.096519

Liu, Ch., Sinkovics, R., Pezderka, N. \& Haghirian P. (2012). Determinants of Consumer Perceptions toward Mobile Advertising - A Comparison between Japan and Austria. Journal of Interactive Marketing, 26(1), 21-32. http:// dx.doi.org/10.1016/j.intmar.2011.07.002

Luo, X. (2002). Uses and gratifications theory and e-consumer behaviors: a structural equation modeling study. Journal of Interactive Advertising, 2(2), 34-41. http://dx.doi.org/10.1080/15252019.2002.10722060

Madan, K. \& Yadav, R. (2018). Understanding and predicting antecedents of mobile shopping adoption: A developing country perspective. Asia Pacific Journal of Marketing and Logistics, 30(1), 139-162. http://dx.doi. org/10.1108/APJML-02-2017-0023

Mahapatra, S. (2017). Mobile shopping among young consumers: an empirical study in an emerging market. International Journal of Retail \& Distribution Management. 45(9), 930-949. https://doi.org/10.1108/ IJRDM-08-2016-0128

Malik, A., Kumra, R. \& Srivastava, V. (2013). Determinants of consumer acceptance of m-commerce. South Asian Journal of Management, 20(2), 102-126. Disponible en: https://search.proquest.com/docview/1429384077?accountid=14777 Consultado: 02/02/2019

Mathieson, K. (1991). Predicting user intentions: comparing the technology acceptance model with the theory of planned behavior. Information Systems Research, 2(3), 173-191. http://dx.doi.org/10.1287/isre.2.3.173

Merisavo, M., Kajalo, S., Karjaluoto, H., Virtanen, V., Salmenkivi, S., Raulas, M. \& Leppäniemi, M. (2007). An empirical study of the drivers of consumer acceptance of mobile advertising. Journal of Interactive Advertising, 7(2), 41-50. http://dx.doi.org/10.1080/15252019.2007.10722130 
Milne,G.R.\& Gordon,M.E.(1993). Direct mail privacy-efficiency trade-offs within an implied social contract framework. Journal of Public Policy and Marketing, 12(2), 206-215.http://dx.doi.org/10.1177/074391569101200206

Natarajan, T., Balasubramanian, S. A. \& Kasilingam, D. L. (2018). The moderating role of device type and age of users on the intention to use mobile shopping applications. Technology in Society. 53, 79 - 90. http://dx.doi. org/10.1016/j.techsoc.2018.01.003

Natarajan, T., Balasubramanian, S. A. \& Kasilingam, D. L. (2017). Understanding the intention to use mobile shopping applications and its influence on price sensitivity. Journal of Retailing and Consumer Services, 37, 8-22. https://doi.org/10.1016/j.jretconser.2017.02.010

Nysveen, H., Pedersen, P. E. \& Thorbjørnsen, H. (2005). Intentions to use mobile services: Antecedents and cross-service comparisons. Journal of the Academy of Marketing Science, 33(3), 330-346. http://dx.doi. org/10.1177/0092070305276149

Oh, L. B. \& Xu, H. (2003). Effects of multimedia on mobile consumer behavior: An empirical study of location-aware advertising. ICIS 2003 Proceedings, 56. Disponible en: https://aisel.aisnet.org/cgi/viewcontent. cgi?article $=1161 \&$ context=icis2003 Consultado: 02/02/2019

Pipitwanichakarn, T., \& Wongtada, N. (2019). Leveraging the technology acceptance model for mobile commerce adoption under distinct stages of adoption. Asia Pacific Journal of Marketing and Logistics. https://doi. org/10.1108/APJML-10-2018-0448

Mafé, C. R., Blas, S. S., \& Tavera $\square$ Mesías, J. F. (2010). A comparative study of mobile messaging services acceptance to participate in television programmes. Journal of Service Management. 21(1), 69-102. https://doi. org $/ 10.1108 / 09564231011025128$

Sanz, S., Martí, J., Ruíz, C. \& Aldás, J. (2011). Determinantes de la aceptación de la publicidad móvil: Actitud hacia la publicidad, control, irritación y utilidad percibida. XXIII Congreso Nacional de Marketing. AEMARK 2011.

Sanz-Blas, S., Ruiz-Mafé, C. \& Martí-Parreño, J. (2015). Message-driven factors influencing opening and forwarding of mobile advertising messages. International Journal of Mobile Communications, 13(4), 339-357. https:// doi.org/10.1504/IJMC.2015.070058

Sirdeshmukh, D., Singh, J. \& Sabol, B. (2002). Consumer trust, value, and loyalty in relational exchanges. Journal of Marketing, 66(1), 15-37. http://dx.doi.org/10.1509/jmkg.66.1.15.18449

Shankar, V., Venkatesh, A. \& Hofacker, C. H., Naik, P. (2010). Mobile Marketing in the Retailing Environment: Current Insights and Future Research Avenues. Journal of Interactive Marketing, 24(2), 111-120. http://dx.doi. org/10.1016/j.intmar.2010.02.006

Scharl, A., Dickinger, A. \& Murphy, J. (2005). Diffusion and success factors of mobile marketing. Electronic Commerce Research and Applications, 4(2), 159-173. http://dx.doi.org/10.1016/j.elerap.2004.10.006

Siau, K. \& Shen, Z. (2003). Mobile communications and mobile services. International Journal of Mobile Communications, 1(1-2), 3-14. http://dx.doi.org/10.1504/IJMC.2003.002457

Stewart, D. W. J. \& Pavlou, P. A. (2002). From consumer response to active consumer: Measuring the effectiveness of interactive media. Journal of the Academy of Marketing Science, 30(4), 376-396. http://dx.doi. org/10.1177/009207002236912

Sun, J., \& Chi, T. (2018). Key factors influencing the adoption of apparel mobile commerce: an empirical study of Chinese consumers. The Journal of The Textile Institute, 109(6), 785-797. https://doi.org/10.1080/00405000. 2017.1371828

Venkatesh, V., Ramesh, V. \& Massey, A. P. (2003). Understanding usability in mobile commerce. Communications of the ACM, 46(12), 53-56. http://dx.doi.org/10.1145/953460.953488

Verkijika, S. F. (2018). Factors influencing the adoption of mobile commerce applications in Cameroon. Telematics and Informatics, 35(6), 1665-1674. https://doi.org/10.1016/j.tele.2018.04.012

Vogt, W. P. (2007). Quantitative research methods for professionals. Allyn and Bacon.

Wang, Y. \& Sun, S. (2010). Examining the role of beliefs and attitudes in online advertising: A comparison between the USA and Romania. International Marketing Review, 27(1), 87-107. http://dx.doi. org/10.1108/02651331011020410 
Watson, R. T., Pitt, L. F., Berthon \& P. Zinkhan, G.M. (2002). U-commerce: expanding the universe of marketing. Journal of the Academy of Marketing Science, 30(4), 333-347. http://dx.doi.org/10.1177/009207002236909

Wong, C. H., Tan, G. W. H., Tan, B. I., \& Ooi, K. B. (2015). Mobile advertising: The changing landscape of the advertising industry. Telematics and Informatics, 32(4), 720-734. https://doi.org/10.1016/j.tele.2015.03.003

Xu, D. J., Liao, S. S. \& Li, Q. (2008). Combining empirical experimentation and modeling techniques: A design research approach for personalized mobile advertising applications. Decision Support Systems, 44(3), 710-724. http://dx.doi.org/10.1016/j.dss.2007.10.002

$\mathrm{Xu}$, D. J. (2006). The influence of personalization in affecting consumer attitudes toward mobile advertising in China. Journal of Computer Information System, 47(2), 9. Disponible en: https://www.tandfonline.com/doi/ab s/10.1080/08874417.2007.11645949 Consultado: 02/04/2019

Zhang, P. (2000). The effects of animation on information seeking performance on the World Wide Web: Securing attention or interfering with primary tasks?. Journal of the Association for Information Systems, 1(1), 1. Disponible en: https://aisel.aisnet.org/jais/vol1/iss1/1/ Consultado: 02/04/2019

Zhang, L., Zhu, J. \& Liu, Q. (2012). A meta-analysis of mobile commerce adoption and the moderating effect of culture. Computers in human behavior, 28(5), 1902-1911. http://dx.doi.org/10.1016/j.chb.2012.05.008Anexo 


\section{Anexo}

Tabla 1

Medición de las variables

\begin{tabular}{|c|c|c|c|c|}
\hline Variable & & Enunciado & Indicador & Fuente \\
\hline \multirow{4}{*}{$\begin{array}{l}\text { Valor utilitarista } \\
\text { percibido (VU) }\end{array}$} & \multicolumn{2}{|c|}{$\begin{array}{l}\text { Los productos que compro en esta aplicación móvil tienen una buena } \\
\text { relación calidad / precio }\end{array}$} & VU1 & $\begin{array}{l}\text { Sirdeshmukh et al. } \\
\text { (2002); Kim y Han }\end{array}$ \\
\hline & \multicolumn{2}{|c|}{$\begin{array}{l}\text { El tiempo que dedico a hacer la compra en esta aplicación móvil } \\
\text { es razonable }\end{array}$} & VU2 & (2011); Kim y Oh (2011) \\
\hline & \multicolumn{2}{|c|}{$\begin{array}{l}\text { El esfuerzo que dedico a hacer la compra en esta aplicación móvil } \\
\text { vale la pena }\end{array}$} & VU3 & \\
\hline & \multicolumn{2}{|c|}{$\begin{array}{l}\text { Creo que, en general, vale la pena utilizar esta aplicación móvil para } \\
\text { comprar }\end{array}$} & VU4 & \\
\hline \multirow{4}{*}{$\begin{array}{l}\text { Utilidad Percibida } \\
\text { (UP) }\end{array}$} & \multirow{4}{*}{$\begin{array}{l}\text { Utilizar esta } \\
\text { aplicación móvil } \\
\text { para comprar... }\end{array}$} & ... hace más fácil el proceso de compra & UP1 & \multirow{8}{*}{$\begin{array}{l}\text { Davis, (1989), Davis et } \\
\text { al. (1989) }\end{array}$} \\
\hline & & $\begin{array}{l}\ldots \text { me permite hacer las compras con más } \\
\text { rapidez }\end{array}$ & UP2 & \\
\hline & & ... me es útil & UP3 & \\
\hline & & $\begin{array}{l}\text {... me permite comprar de una manera más } \\
\text { eficiente }\end{array}$ & UP4 & \\
\hline \multirow{4}{*}{$\begin{array}{l}\text { Facilidad de uso } \\
\text { percibida (FU) }\end{array}$} & \multirow{4}{*}{$\begin{array}{l}\text { Utilizar esta } \\
\text { aplicación móvil } \\
\text { para comprar... }\end{array}$} & ... ha sido fácil de aprender para mi & FU1 & \\
\hline & & $\ldots$ me resulta sencillo & FU2 & \\
\hline & & ... no requiere mucho esfuerzo mental & FU3 & \\
\hline & & $\begin{array}{l}\ldots \text { es fácil siguiendo las instrucciones } \\
\text { disponibles en la aplicación móvil }\end{array}$ & FU4 & \\
\hline \multirow{4}{*}{$\begin{array}{l}\text { Valor informativo } \\
\text { (VI) }\end{array}$} & \multirow{4}{*}{$\begin{array}{l}\text { Las alertas móviles } \\
\text { que recibo... }\end{array}$} & $\begin{array}{l}\text {... me proveen de información oportuna } \\
\text { sobre los productos disponibles }\end{array}$ & VI1 & \multirow[t]{4}{*}{$\begin{array}{l}\text { Wang y Sun (2010), Lui } \\
\text { et al. (2012) }\end{array}$} \\
\hline & & $\begin{array}{l}\text {... me suministran de información relevante } \\
\text { sobre los productos disponibles }\end{array}$ & VI2 & \\
\hline & & $\begin{array}{l}\text {... son una buena fuente de información } \\
\text { sobre los productos disponibles }\end{array}$ & VI3 & \\
\hline & & $\begin{array}{l}\text {... contienen información actualizada sobre } \\
\text { los productos disponibles }\end{array}$ & VI4 & \\
\hline \multirow{2}{*}{$\begin{array}{l}\text { Control percibido } \\
\text { (CP) }\end{array}$} & \multirow[t]{2}{*}{$\begin{array}{l}\text { Siento que con esta } \\
\text { aplicación móvil... }\end{array}$} & $\begin{array}{l}\text {... puedo elegir el tipo de alertas que recibo } \\
\ldots \text { puedo controlar fácilmente el número de } \\
\text { alertas que recibo }\end{array}$ & $\begin{array}{l}\mathrm{CP} 1 \\
\mathrm{CP} 2\end{array}$ & \multirow[t]{2}{*}{$\begin{array}{l}\text { Kautonen y Kohtamaki } \\
(2006)\end{array}$} \\
\hline & & $\begin{array}{l}\text {... puedo cancelar fácilmente el permiso } \\
\text { para enviarme alertas }\end{array}$ & $\mathrm{CP} 3$ & \\
\hline \multirow{2}{*}{$\begin{array}{l}\text { Personalización } \\
\qquad(\mathrm{PE})\end{array}$} & \multirow{2}{*}{\multicolumn{2}{|c|}{$\begin{array}{l}\text { Las alertas móviles me muestran mensajes personalizados } \\
\text { Los contenidos de las alertas móviles se ajustan a mis preferencias } \\
\text { e intereses }\end{array}$}} & PE1 & \multirow{2}{*}{$\begin{array}{l}\text { Xu, (2006), Merisavo et } \\
\text { al. (2007) }\end{array}$} \\
\hline & & & PE2 & \\
\hline \multirow{3}{*}{ Irritación (IR) } & \multirow{3}{*}{$\begin{array}{l}\text { El servicio de alertas } \\
\text { móviles... }\end{array}$} & ... es ofensivo & IR1 & \multirow[t]{3}{*}{ Lee et al. (2006) } \\
\hline & & ... es molesto & IR2 & \\
\hline & & ... es intrusivo & IR3 & \\
\hline
\end{tabular}

